\title{
Evolution Temporelle Et Spatiale De La Macroflore Benthique De L'ecosysteme Lagunaire De Nador
}

\author{
Najih Mohamed, Dr. \\ Berday Nadia, Dr. \\ Institut Agronomique et Vétérinaire Hassan II-Rabat-Morocco \\ Rezzoum Nor-dine, Dr. \\ Institut National de Recherche Halieutique -Casablanca- Morocco \\ Mounir Fouad, Dr. \\ Ecole Nationale Forestière des Ingénieurs - Salé-Morocco
}

Lamrini Abdeljaouad, Dr.

Institut Agronomique et Vétérinaire Hassan II-Rabat

Nachite Driss, Dr.

Université Abdelmalek Essaadi Faculté des Sciences de Tétouan-Morocco

doi: 10.19044/esj.2016.v12n36p355 URL:http://dx.doi.org/10.19044/esj.2016.v12n36p355

\begin{abstract}
The objective of the present study undertaken in 2011is to evaluate the status of the benthic macroflora of the Nador lagoon after the management works carried out in 2009 to improve the lagoon environmental quality.

Relying on a previous study carried out in 1987, the results of analysis in 2011 showed that the lagoon seabed macroflora recorded a marked decline and an overall change in its distribution and specific composition. The marine Phanerogams, represented by Cymodocea and Zostera, in association with Caulerpa, which were the main vegetationin 1987, were replaced by a macroflora highly dominated by macroalgae (64.3\% of the total area), essentially represented by Rhodophyceae (58\% of total area).In 2011, the genus Alsidiumwas the main representative in the macroflora(55\% of total area), while the genus Caulerpa, and Phanerogamssaw their area of occurrence regress to $25.6 \%$ and $18 \%$ respectively.
\end{abstract}

Keywords: Nador lagoon - Structure of the benthic macroflora - Management -Facies -Physicochemical characteristics.

\section{Résumé}

La présente étude, réalisée en 2011, a pour objectif de faire établir la nouvelle structure de la macroflore benthique de l'écosystème lagunaire de 
Nador, après les travaux d'aménagement entrepris en 2009 pour l'amélioration de la qualité environnementale de la lagune,

Par référence à une étude antérieure réalisée en 1987, les résultats d'analyse ont permis de mettre en en évidence que la macroflore benthique au niveau de la lagune a enregistré une régression et un changement global de sa distribution et de sa composition spécifique. Les Phanérogames marines (du genre Cymodocea et Zostera), en association avec le genre Caulerpa qui constituaient la végétation principale de la lagune en 1987, ont été remplacés par une macroflore hautement prédominée par les macroalgues $(64,3 \%$ de la superficie totale).

En 2011, le genre Alsidium constituait le principal représentant de la macroflore (55\% de la surface totale) Par contre, le genre Caulerpa et les Phanérogames ont vu leurs surfaces d'occupation régresser à $25,6 \%$ et $18 \%$ respectivement.

Mots clés : Lagune de Nador - Structure de la macroflore benthique Aménagement - Faciès- Caractéristiques physicochimiques.

\section{Introduction}

La lagune de Nador (ou Marchica ou Sebkha Bou Areg) est un écosystème lagunaire situé dans la partie nord orientale de la côte méditerranéenne du Maroc et qui s'étend sur $25 \mathrm{~km}$ de longueur et 0,3 à 1,7 $\mathrm{km}$ de largeur. Avant 2009, la lagune communiquait avec la mer par intermittence, par l'intermédiaire d'une passe naturelle. Par la suite, et depuis 2009, dans le cadre du plan d'aménagement de la lagune pour l'amélioration de sa qualité environnementale, la communication a été fermée et remplacée par un nouveau chenal plus large. De même qu'une station d'épuration de type boues activées a été mise en place afin de desservir la région de Nador dans le but de réduire la pollution au niveau de la lagune.

La lagune de Nador est considérée comme un site de grand intérêt biologique et écologique (SIBE). Ce site est également le siège d'un certain nombre d'activités économiques dont la pêche, l'aquaculture, le tourisme et l'agriculture. De ce fait, il est soumis à une forte pression anthropique. Avant 2009, la situation était d'avantage aggravée par les décharges d'eaux usées brutes d'origine urbaine et industrielle face à un faible taux de renouvellement et suite à des colmatages réguliers de la seule passe qui communiquait avec la mer.

Les impacts sur la qualité environnementale de la lagune ont été fortement ressentis, entraînant une détérioration de la qualité des eaux et du sédiment et par conséquent une modification de la distribution de la macroflore benthique. Des études entreprises entre 1979 et 1997 ont montré que la structure de la macroflore a connu des changements importants au cours 
de cette période. Ainsi, jusqu'en 1979, le fond lagunaire était quasiment tapissé par des prairies de Zostères et de posidonies (Saubade, 1979), remplacées à partir des années quatre-vingt, par un herbier mixte à Cymodocea nodosa et Caulerpa proliferaqui colonisait toute la zone centrale, alors que le genre Ruppia était limité aux deux extrémités confinées de la lagune (Guelorget, 1987). Par la suite et jusqu'en1997, la répartition de la macroflore benthique est restée sensiblement la même (Lefebvre et al., 1997).

Les travaux d'aménagement en 2009 ont aboutis à des changements importants de la qualité de l'eau et de la distribution des sédiments au niveau de la lagune résultant de la modification du régime hydrologique et de la réduction de la décharge qui la polluait (Najih et al. 2016). Ces changements ont certainement eu un effet sur la structure de la macroflore benthique. Ainsi, le présent travail consiste à faire établir la nouvelle structure de la macroflore benthique de la lagune de Nador après les travaux d'aménagement, à travers une analyse de sa composition et la cartographie de sa nouvelle distribution.

\section{Matériels et méthodes}

\section{Situation géographique et morphologie de la lagune}

La lagune de Nador ( $\left.34^{\circ} 54^{\prime} \mathrm{N}-35^{\circ} 17^{\prime} \mathrm{N} ; 02^{\circ} 10^{\prime} \mathrm{W}-03^{\circ} 05^{\prime} \mathrm{W}\right)$, est située dans la partie NE de la façade méditerranéenne marocaine. Elle est limitée au NO par Le Cap des Trois Fourches et au SE par le Cap de 1'Eau, du côté marin par le cordon dunaire et du côté continental par le massif du Cap de Trois Fourches, le volcan du Gourougou, le massif de Béni-Bou-Ifrour, la plaine de Bou-Areg et le piedmont septentrional des Kebdana (figure 1a).La lagune de forme semi-elliptique est séparée par le pointèrent volcanique de la colline d'Atalayoun, en deux bassins de dimensions inégales (petit bassin au $\mathrm{N}$, sur la rive continentale et grand bassin qui constitue la majeure partie de la lagune) (figure $1 \mathrm{~b}$ ).

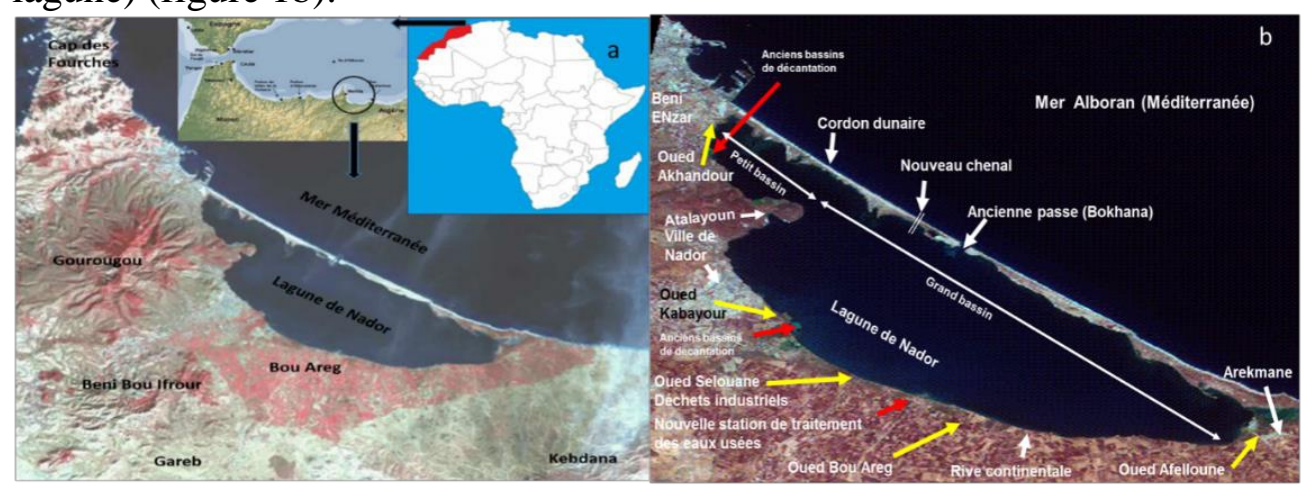

Figure 1 : Situation géographique de la lagune de Nador et de son bassin versant (bassin versant (a), réseau hydrographique et morphologie de la lagune (b) 
Compte tenu de cette forme géomorphologique et en référence aux travaux antérieurs, la lagune peut être divisée en 4 zones : Zone I (extrême NW ou, petit bassin) et Zone II (extrême SE du grand bassin) sont considérés comme les zones les plus confinées de la lagune. La Zone III (partie sud, face au continent) est la plus influencée par les apports anthropiques et d'eau douce. La Zone IV (partie nord, face au cordon dunaire) est la plus influencée par les apports d'eau de mer à travers le nouveau chenal et par infiltration à travers le banc de sable.

A partir de 2009, la lagune communique avec la mer par l'intermédiaire d'un chenal artificiel qui traverse le cordon dunaire à $1,5 \mathrm{~km}$ au nord du Bokhana (l'ancienne passe). Ce chenal (35 $\left.25^{\prime} \mathrm{N}, 02^{\circ} 86^{\prime} \mathrm{O}\right)$ se compose de deux brise-lames de longueurs de $1450 \mathrm{~lm}$ à $1^{\prime}$ 'Est et $1.350 \mathrm{~lm}$ à l'Ouest, et d'une profondeur de 6,00 m NGM et sont séparés par une largeur de $300 \mathrm{~lm}$ (Marchicamed, 2010). L'ouverture du nouveau chenal a contribué à l'accroissement des échanges d'eau entre la mer et la lagune, et à l'amélioration de la qualité environnementale de cette dernière (Najih et al, 2016).

\section{Caractéristiques climatologiques de la lagune}

La zone de Nador présente un climat de type méditerranéen. Il se distingue par la succession de deux saisons contrastées (Dakki, 2003) : un été chaud et sec, s'étalant de juin à octobre avec une température moyenne de $20^{\circ} \mathrm{C}$, et une période fraîche et pluvieuse de Novembre à Mai, avec des températures moyennes de $12^{\circ} \mathrm{C}$. Les précipitations annuelles varient de 224 $\mathrm{mm} / \mathrm{an}$ à $390 \mathrm{~mm} / \mathrm{an}$ ( $\mathrm{Re}, 2010)$. Le régime général des vents est WSW $(24,8 \%)$ de novembre à mai, et ENE (32,9\%) de Mai à Octobre avec des intensités moyennes variantes entre 3,8 à $6,2 \mathrm{~m} / \mathrm{s}$ (Hilmi, 2005).

\section{Réseau hydrologique et dynamique sédimentaire de la lagune}

Les eaux de la lagune ont deux origines, l'une continentale et l'autre marine. Avant 2009, des périodes de sécheresse et/ou de colmatage de la passe ont conduit à un déficit hydrique, qui a été plus ou moins aigu, en fonction de l'intensité de ces deux phénomènes. Du côté continent, la lagune est alimentée par la nappe phréatique et par certains émissaires, à écoulement temporaire, de la bordure continentale (figure 1b).

L'évolution du faciès sédimentaire de la lagune entre 1987 et 2011 est marquée essentiellement par un rétrécissement de l'étendue des fonds vasosableux et un élargissement de celle des fonds vaseux. Le sable fin qui couvrait le cordon dunaire s'est limité à ses deux extrémités NO et SE (Najih et al., 2016) (figure 2). 


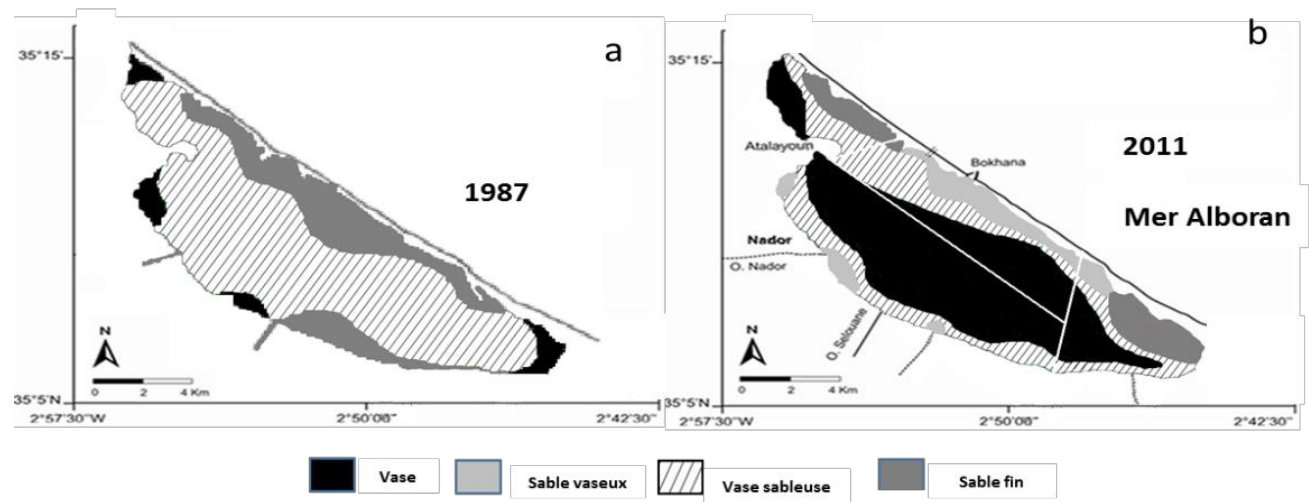

Figure 2 : Evolution du faciès sédimentaire de la lagune de Nador entre 1987 (a) (Guelorget et al., 1987) et 2011 (b) (Najih et al., 2016)

\section{Bathymétrie et circulation hydrodynamique de la lagune}

La lagune reste en général peu profonde, dépassant à peine les $7 \mathrm{~m}$ (figure 3a). La majorité du plan d'eau $(70 \%)$ présente une profondeur ne dépassant pas les $5 \mathrm{~m}$ (Hilmi, 2005, 2015). Le moteur de la circulation marine des eaux au sein de la lagune est le vent. Les courants ont une très grande influence dont les intensités sont supérieures à $1 \mathrm{~m} / \mathrm{s}$ en périodes de vives eaux. Ils diminuent d'intensité au centre $(<0,5 \mathrm{~m} / \mathrm{s})$ et en bordure des rives continentales de la lagune $(<0,3 \mathrm{~m} / \mathrm{s})$ (figure $3 b)$. Les courants du fond de directions inverses présentent des intensités faibles $(<0,2 \mathrm{~m} / \mathrm{s})$ (Hilmi, 2015).
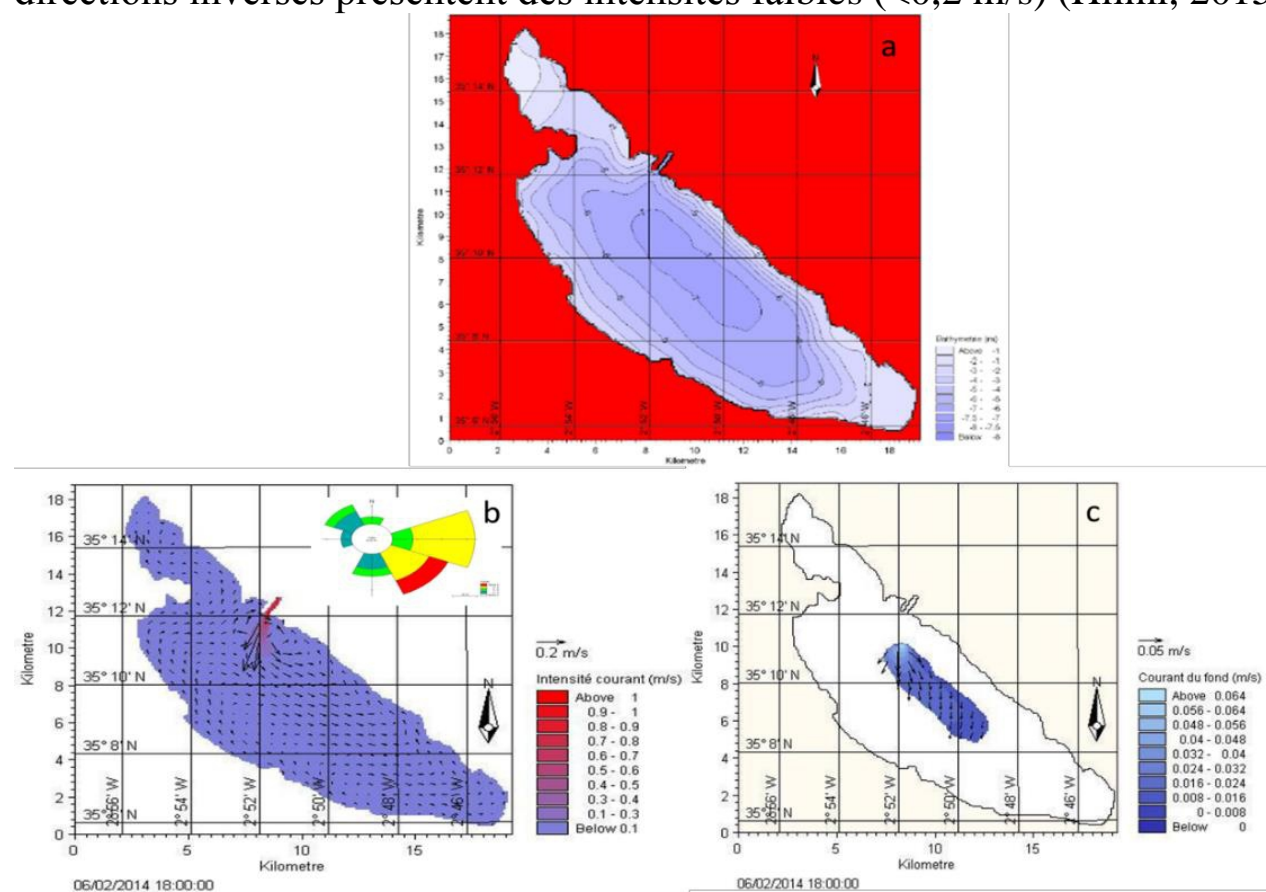

Figure 3 : Bathymétrie (a) et Circulation hydrodynamique de surface (b) et de fond (c) de la lagune de Nador (Hilmi, 2015) 


\section{Paramètres physico-chimiques de l'eau de la lagune}

L'étude de la structure et de la répartition spatiale de la flore benthique a été menée en parallèle avec un suivi des paramètres physico-chimiques de l'eau essentiels à la croissance de la macroflore. Les données relatives à ce suivi sont consignées dans le tableau 1. Elles montrent une différence de répartition de ces paramètres entre les quatre zones, particulièrement pour les nutriments ( $\mathrm{N}$ et $\mathrm{P})$. Les eaux de la zone I semblent être plus riches en nutriments, avec des teneurs en oxygène dissous (OD) plus faibles. La zone III est également plus riche en nutriments que la zone IV, mais présente des teneurs en OD assez proches de celles de la zone IV. La zone II semble être moins riche en OD au niveau du fond. De ce fait, les zones I et III semblent avoir un niveau d'eutrophisation plus avancé que celui de la zone IV.

Tableau 1 : Teneurs moyennes et écart-types des paramètres physico-chimiques de l'eau dans la lagune de Nador (Najih et al., Non publié).

\begin{tabular}{|c|c|c|c|c|c|}
\hline & & Zone I & Zone II & Zone III & Zone IV \\
\hline pH & $\begin{array}{l}\text { surfac } \\
\mathrm{e}\end{array}$ & $8,41 \pm 0,04$ & $8,35 \pm 0,02$ & $8,28 \pm 0,03$ & $8,32 \pm 0,04$ \\
\hline \multirow{2}{*}{ Salinité \%o } & $\begin{array}{l}\text { surfac } \\
\mathrm{e}\end{array}$ & $37,87 \pm 0,44$ & $\begin{array}{c}37,77 \pm \\
0,04\end{array}$ & $36,99 \pm 0,28$ & $37,15 \pm 0,08$ \\
\hline & Fond & $38,27 \pm 0,18$ & $\begin{array}{c}37,83 \pm \\
0,11\end{array}$ & $36,13 \pm 0,23$ & $37,07 \pm 0,17$ \\
\hline \multirow{2}{*}{$\begin{array}{c}\text { Température } \\
\left({ }^{\circ} \mathrm{C}\right)\end{array}$} & $\begin{array}{l}\text { surfac } \\
\mathrm{e}\end{array}$ & $26,93 \pm 0,58$ & $27,9 \pm 0,53$ & $27,47 \pm 0,87$ & $25,65 \pm 0,72$ \\
\hline & Fond & $27,23 \pm 0,51$ & $\begin{array}{c}27,77 \pm \\
0,38 \\
\end{array}$ & $26,01 \pm 1,64$ & $24,1 \pm 1,77$ \\
\hline \multirow[t]{2}{*}{ Oxygène (mg/l) } & $\begin{array}{c}\text { surfac } \\
\mathrm{e}\end{array}$ & $8,33 \pm 1,11$ & $6,33 \pm 0,89$ & $8,11 \pm 1,21$ & $7,92 \pm 1,7$ \\
\hline & Fond & $6,7 \pm 0,3$ & $4,5 \pm 0,5$ & $7,15 \pm 0,95$ & $7,7 \pm 1,64$ \\
\hline \multirow{2}{*}{ Nitrites $(\mu \mathrm{g} / \mathrm{l})$} & $\begin{array}{c}\text { surfac } \\
\mathrm{e}\end{array}$ & $9,71 \pm 4,6$ & $\begin{array}{c}12,27 \pm \\
2,04 \\
\end{array}$ & $13,29 \pm 4,97$ & $2,3 \pm 0,77$ \\
\hline & Fond & $5,62 \pm 0,51$ & $\begin{array}{c}11,76 \pm \\
6,64\end{array}$ & $9,37 \pm 6,3$ & $8,69 \pm 2,56$ \\
\hline \multirow{2}{*}{ Nitrates $(\mu \mathrm{g} / \mathrm{l})$} & $\begin{array}{l}\text { surfac } \\
\text { e }\end{array}$ & $22,49 \pm 9,94$ & $\begin{array}{c}60,42 \pm \\
7,26\end{array}$ & $\begin{array}{c}129,56 \pm \\
75,73\end{array}$ & $\begin{array}{c}131,87 \pm \\
8,49\end{array}$ \\
\hline & Fond & $\begin{array}{c}413,34 \pm \\
20,29 \\
\end{array}$ & $\begin{array}{c}13,95 \pm \\
7,76 \\
\end{array}$ & $85,96 \pm 86,89$ & $63,55 \pm 5,89$ \\
\hline \multirow{2}{*}{$\begin{array}{c}\text { Ammonium } \\
(\mu \mathrm{g} / \mathrm{l})\end{array}$} & $\begin{array}{c}\text { surfac } \\
\mathrm{e}\end{array}$ & $3,94 \pm 1,69$ & $25,13 \pm 4,5$ & $93,54 \pm 6,64$ & $43,13 \pm 6,25$ \\
\hline & Fond & $47,81 \pm 5,06$ & $19,13 \pm 5,5$ & $7,8 \pm 2,25$ & $6,31 \pm 5,7$ \\
\hline \multirow[t]{2}{*}{$\begin{array}{c}\text { Azote minéral } \\
(\mu \mathrm{g} / \mathrm{l})\end{array}$} & $\begin{array}{c}\text { surfac } \\
\mathrm{e}\end{array}$ & $12,05 \pm 20,29$ & $32,61 \pm 5,5$ & $78,8 \pm 15,5$ & $59,1 \pm 3,5$ \\
\hline & Fond & $155,6 \pm 8,62$ & $14,95 \pm 4,5$ & $34,38 \pm 13,5$ & $26,18 \pm 4,3$ \\
\hline $\begin{array}{c}\text { Ortophosphates } \\
(\mu \mathrm{g} / \mathrm{l})\end{array}$ & $\begin{array}{l}\text { surfac } \\
\mathrm{e}\end{array}$ & $264,9 \pm 13,83$ & $\begin{array}{c}40,19 \pm \\
6,54\end{array}$ & $43,85 \pm 19,49$ & $51,88 \pm 4,8$ \\
\hline
\end{tabular}




$$
\begin{array}{lcccc}
\text { Fond } & 80,38 \pm 7,31 & \begin{array}{c}
40,19 \pm \\
5,23
\end{array} & 56,63 \pm 1,83 & 62,12 \pm 4,62
\end{array}
$$

\section{Méthode d'échantillonnage de la macroflore benthique}

L'échantillonnage est effectué au niveau de 250 stations régulièrement reparties d'une manière aléatoire sur l'ensemble de la lagune (figure 4). Pour permettre de comparer nos résultats avec ceux avant l'aménagement, le réseau d'échantillonnage choisi est pratiquement celui qui a été adopté par Id Hala et al. (2004 et 2005). Les prélèvements ont été effectués, au cours de quatre campagnes entre mars et juin 2011, par plongée sous-marine.

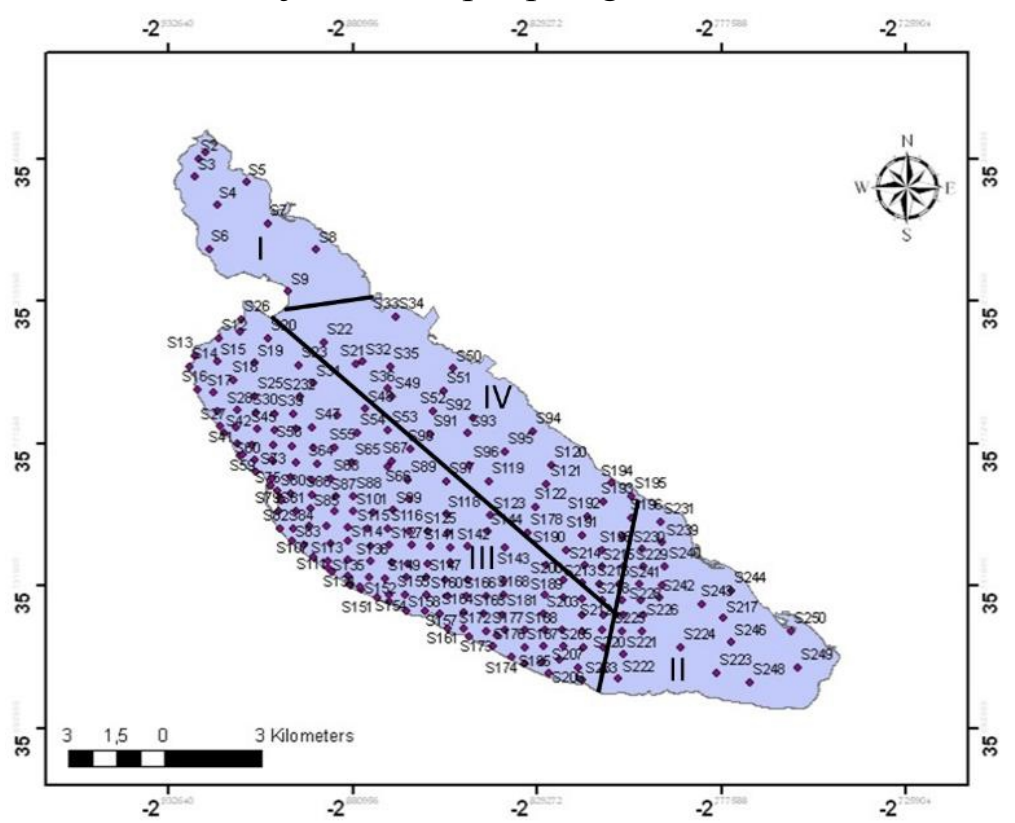

Figure 4 : Réseau d'échantillonnage de la macroflore benthique de la lagune de Nador

La surface du fond de chaque station de prélèvements est fixée à 0,25 $\mathrm{m}^{2}$ et ce, par l'utilisation d'un quadrat métallique de $(50 \mathrm{~cm} \times 50 \mathrm{~cm})$. La totalité de la flore fixée sur le substrat, se trouvant à l'intérieur de ce quadrat est prélevée.

\section{Méthode d'analyse}

\section{Intégration du taux de recouvrement des échantillons}

Etant donné la répartition hétérogène du tapis végétal à la surface du sol de la lagune d'une part et la faible représentativité du quadrat d'échantillonnage (surface : $0,25 \mathrm{~m}^{2}$ ) d'autre part, il a été indispensable d'introduire la notion du taux de recouvrement (TR) pour que les données de mesure soient extrapolables à une échelle plus importante lors de la cartographie (Canfield, 1942). 
Le taux de recouvrement d'un groupe, ou d'une espèce (TRi) est la projection verticale de sa surface occupée par rapport à la surface totale. Il est exprimé en pourcentage et déterminé par la méthode de la ligne d'interception (Canfield, 1942). Pour notre cas, ce taux est approché d'une manière visuelle par la plongée, en prospectant la station d'échantillonnage.

\section{Identification des espèces de la macroflore benthique}

L'identification des taxons de la macroflore s'est basée sur les caractères macroscopiques tels que la couleur, la forme et la taille en utilisant les fiches d'identification des espèces FAO (Fischer, W., M.-L. Bauchot et M. Schneider, 1987). Pour la majorité des spécimens, l'identification a été limitée au genre, seules deux espèces ont été identifiées :Rytiphlea tinctoriaet Caulerpa prolifera. Pour les Phéophycées, seul le genre Cystoseiraa été identifié.

\section{Cartographie des champs de la macroflore benthique}

Pour mettre en évidence la structure spatiale des gisements, les informations de chaque station échantillonnée, en particulier la position en coordonnée géographique (latitude, longitude), la présence ou absence de la flore et le taux de recouvrement, ont été intégrées en système d'information géographique SIG de Arc-View. Cette intégration permet de localiser les points de prospection et de réaliser les traitements numériques nécessaires pour la cartographie et le calcul de la surface de chaque répartition de chaque spécimen de la macroflore. Le système de projection choisi à cet effet, est celui de LAMBERT-LCC (Lambert Conique Conforme) dont les paramètres sont définis dans le système de référence géodésique CLARKES 1880.

\section{Analyse statistique}

En plus des calculs des moyennes et des écart-types moyens des paramètres mesurés, la répartition spatiale a été identifiée par interpolation des données de mesure ponctuelle sur toute la lagune pour une grille de mesure à maille carrée de $20 \mathrm{~m}$ x $20 \mathrm{~m}$ de taille. L'estimation des valeurs des points non échantillonnés a été approchée par l'application du concept du plus proche voisin, basé sur la méthode de l'inverse de la distance (Inverse Weighted Distance IWD).

\section{Résultats}

\section{Répartition spatiale de la macroflore benthique}

La répartition spatiale de la macroflore benthique est présentée dans les figures 5 et 6 . Les résultats montrent que la macroflore benthique couvre $64,3 \%$ de la superficie totale de la lagune, avec une grande variabilité de recouvrement, allant de $0 \%$ à $100 \%$. La macroflore est hautement prédominée par les 
macroalgues qui couvrent $63,8 \%$ de la surface totale de la lagune, tandis que les phanérogames se limitent à $17 \%$ de la surface totale.

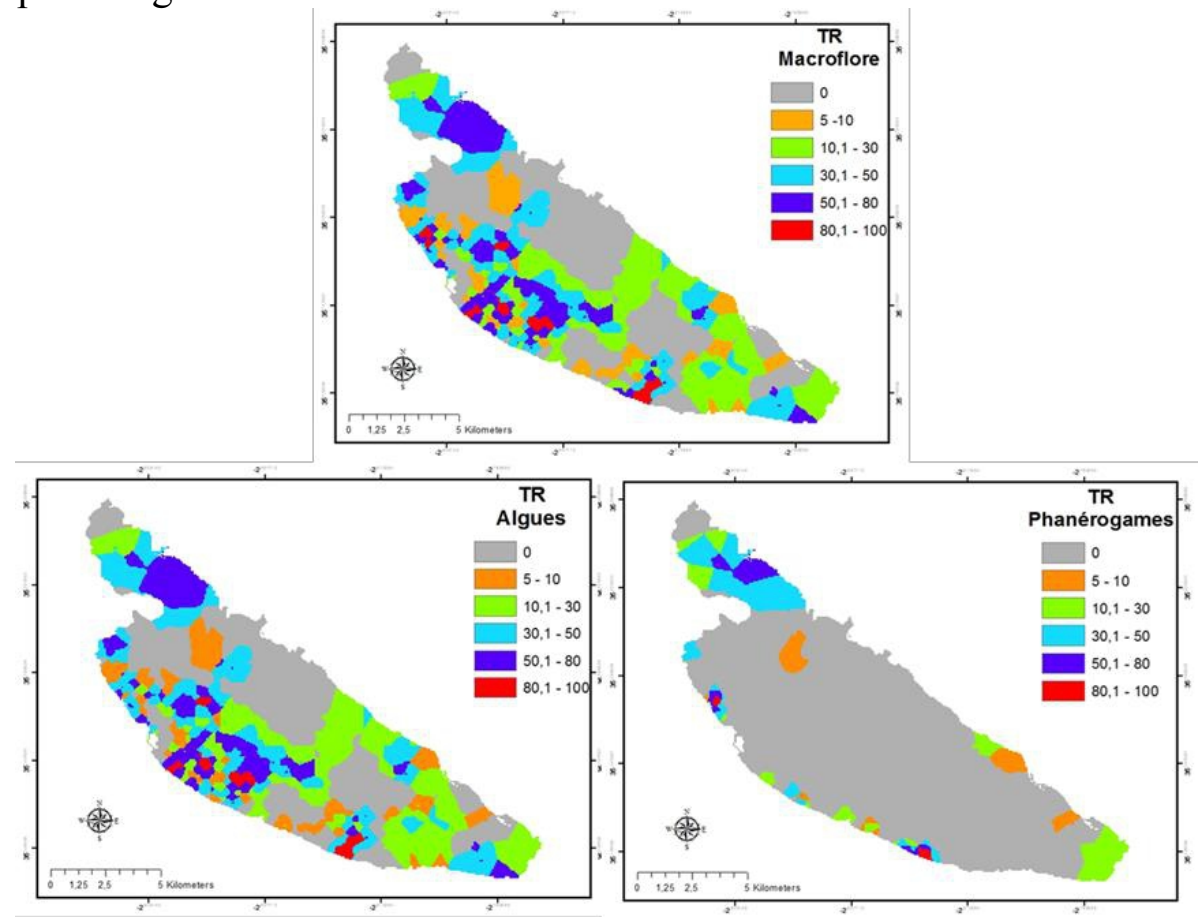

Figure 5 : Répartition spatiale de la macroflore benthique totale (a) des macroalgues (b) et des phanérogames marines (c)

Le taux d'occupation spatiale de la macroflore benthique par classe de taux de recouvrement (TR), au niveau de la lagune est comme suit : les TR de l'ordre de $80-100 \%$ occupent moins que $2 \%$ de la surface totale, les TR de 50 à $80 \%$ occupent $12,1 \%$ de la surface totale, les TR de $30-50 \%$ occupent $18,5 \%$ de la surface totale, les TR de $10-30 \%$ occupent $23,3 \%$ de la surface totale et les TR de 5-10\% occupent $8,7 \%$ de la surface totale (figure 6 ).

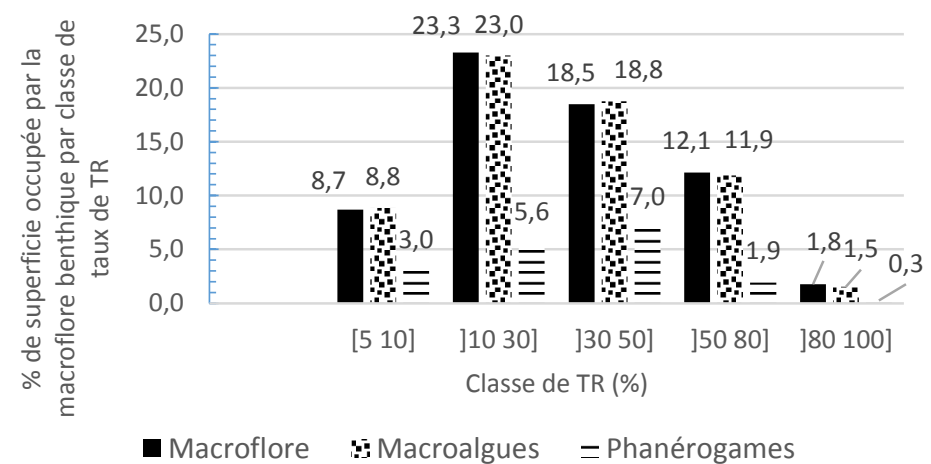

Figure 6 : Taux d'occupation spatiale de la macroflore benthique par classe de taux de recouvrement (TR) au niveau de la lagune de Nador 
La répartition de la macroflore benthique est beaucoup moins importante dans la zone IV où elle se présente avec de faibles TR dans la partie SE et reste quasi-absente dans partie NO.

Par référence à la figure $2 b$, on constate que la macroflore benthique colonise des fonds à faciès variées à l'exception des fonds sablo-vaseux où la végétation reste absente ou très faible. En général, ce sont les fonds vaseux qui supportent la majeure partie de la macroflore. Les TR les plus importants ont été particulièrement observés dans des aires à fonds vaseux et vaso-sableux de la zone III, surtout le long de la rive continentale, autour des bassins de lagunage et de la station d'épuration. Les macroalgues, hautement prédominantes dans la macroflore de la lagune, suivent globalement la même répartition que celle de la macroflore. Elles montrent un maximum de concentration au niveau de la zone III, avec des TR très variables généralement supérieurs à $30 \%$.

Les phanérogames marines sont moins représentées au niveau de la lagune. Leur fréquence de présence au niveau des échantillons ne dépasse pas $13 \%$ et restent cantonnées dans des zones très limitées de la lagune, particulièrement au niveau du petit bassin (zone I), la bordure continentale et la partie SE de la bordure marine de la lagune.

\section{Répartition des principaux groupes taxonomiques des macroalgues benthiques de la lagune}

Trois groupes taxonomiques d'algues marines sont rencontrés dans la lagune de Nador: les Rhodophycées (3 taxons), Chlorophycées (4 taxons) et les Phéophycées (1 taxon).

La répartition des principaux groupes taxonomiques des macroalgues benthiques de la lagune est présentée sur la figure 7 .

\section{Classe des Rhodophycées}

La classe des Rhodophycées est le groupe systématique le plus représenté en matière de répartition spatiale au sein de la lagune, qui suit la même tendance de la répartition de l'ensemble des algues (figures 5 et 7). Elle est composée par trois espèces: Alsidium sp. Gracilaria sp., et Rytiphlea tinctoria. 


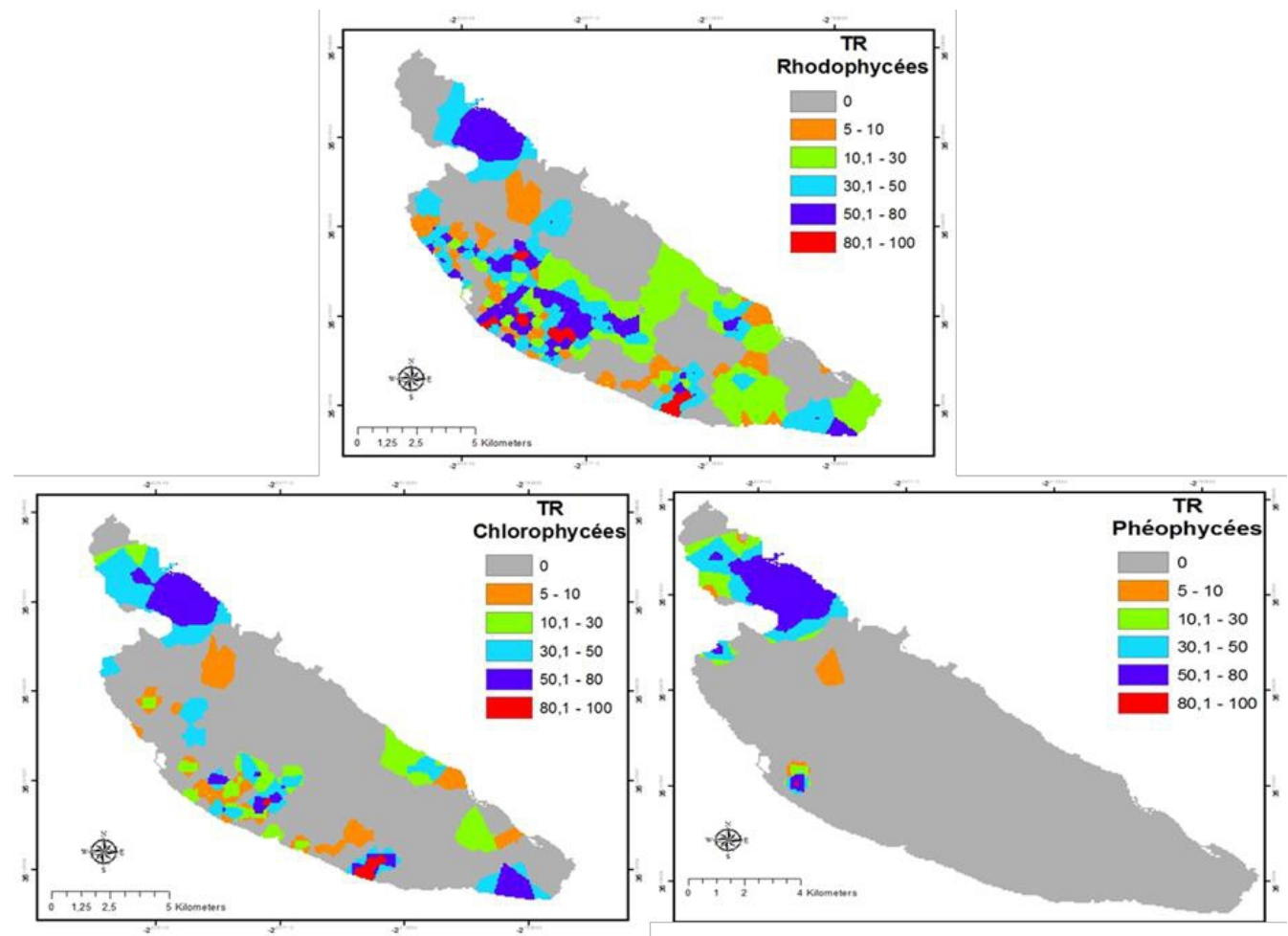

Figure 7 : Répartition spatiale des principales classes de macroalgues au sein de la lagune de Nador: Rhodophycées (a), Chlorophycées (b) et Phéophycées (c)

Cette classe occupe environ 58\% de la surface de la lagune avec des TR qui varient entre 0 et $100 \%$ et une moyenne de $(23,1 \% \pm 5,7 \%)$. La majeure partie des zones I, II et IV n'est pas couverte par les Rhodophycées. Le recouvrement est plus important dans la zone III, particulièrement en face de la ville de Nador et le long des anciens bassins de décantation de la station d'épuration. Selon le taux d'occupation de la lagune par les Rhodophycées, $12,5 \%$ de la superficie de la lagune présentent un TR qui dépasse les $50 \%$ avec $1,5 \%$ seulement de la superficie ayant un TR qui dépasse $80 \%, 17,5 \%$ de la superficie présentent un TR de 30 à $50 \%, 20 \%$ de la superficie un TR de 10 à $30 \%$ et $8 \%$ de la superficie un TR de 5 à $10 \%$ (figure 8 ). 


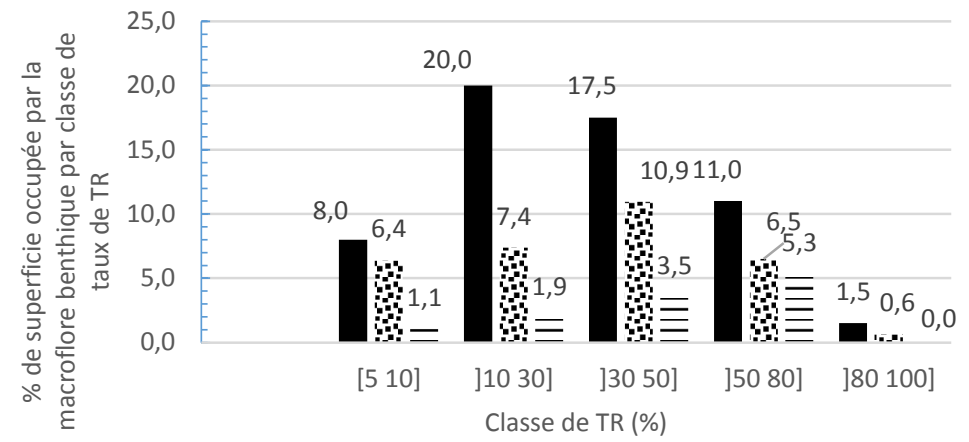

- Rhodophycées : Chlorophycées = Phéophycées

Figure 8 : Taux d'occupation spatiale des principaux groupes des macroalgues au sein de la lagune de Nador

\section{Classe des Chlorophycées}

La classe des Chlorophycées constitue le 2ème groupe après les Rhodophycées en matière d'importance de répartition. Elle est constituée par les espèces suivantes : Caulerpa prolifera, Chaetomorpha sp., Enteromorpha $s p$. et Uulva sp. Cette classe se répartit sur 31,8\% de la superficie de la lagune, avec une fréquence de présence au niveau des échantillons de $24,3 \%$. Le TR moyen est de $9,7 \% \pm 23,8 \%$. Hormis certains endroits, la répartition de ces algues se fait d'une manière irrégulière. Elles sont éparpillées sur certaines localités de la rive continentale de la zone III, alors qu'elles sont bien réparties dans la zone I. Elles sont pratiquement absentes dans les zones profondes de la lagune et dans la zone VI et très faiblement représentées dans la zone II.

Tous les faciès de la lagune constituent des supports pour les Chlorophycées. Toutefois, leur répartition reste limitée à des profondeurs ne dépassant pas $5 \mathrm{~m}$. Les Chlorophycées sont presque réparties exclusivement sur le rivage (à quelques exceptions).

\section{Classe des Phéophycées}

La classe des Phéophycées est faiblement représentée dans la lagune de Nador (11,7\% de la superficie totale). Ce groupe est cantonné quasiment au nord-ouest (de la zone I) et sur des espaces très limités, au milieu de la lagune en face du chenal (de la zone IV) et au niveau de la rive continentale près des anciens bassins de décantation dans la zone III (figure 7). Le TR varie de 0 à $100 \%$, avec 5,3\% de la superficie couverte par les Phéophycées avec un TR de $50-80 \%, 3,5 \%$ avec un TR de $30-50 \%$ et $3 \%$ avec un TR inférieur à $30 \%$ (figure 8 ). 


\section{Répartition des différents taxons d'algues et de Phanérogames marines Répartition des taxons selon la nature du faciès sédimentaire}

La répartition des différents taxons d'algues et de Phanérogames semble étroitement liée à la nature du faciès sédimentaire du fond de la lagune. Le tableau 2 illustre la réparation des différents taxons en fonction du faciès sédimentaire.

Tableau 2 : Répartition des différents taxons de la macroflore benthique en fonction du faciès sédimentaire de la lagune

\begin{tabular}{|c|c|c|c|c|c|}
\hline \multirow[t]{2}{*}{ Groupe d'espèces } & \multirow[t]{2}{*}{ Genre/espèce } & \multicolumn{4}{|c|}{ Faciès } \\
\hline & & 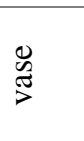 & 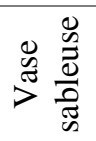 & 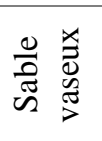 & $\frac{\ddot{\theta}}{\tilde{\tilde{D}}}$ \\
\hline \multirow[t]{3}{*}{ Rhodophycées } & Alsidium & +++ & +++ & + & + \\
\hline & Gracilaria & o & +++ & +++ & + \\
\hline & Rytiphlea & +++ & +++ & + & + \\
\hline \multirow[t]{4}{*}{ Chlorophycées } & Caulerpa & +++ & +++ & +++ & +++ \\
\hline & Chaetomorpha & +++ & +++ & $\mathrm{o}$ & +++ \\
\hline & Ulva & o & + & o & +++ \\
\hline & Enteromorpha & o & + & o & o \\
\hline Phéophycées & Cystoseria & o & + & o & + \\
\hline \multirow[t]{2}{*}{ Phanérogames marines } & Cymodocea & + & +++ & + & ++ \\
\hline & Zostera & o & +++ & o & +++ \\
\hline
\end{tabular}

$\mathrm{o}:$ absent $+++:$ forte présence ++ moyenne présence + faible présence

\section{Répartition des espèces de Rhodophycées}

L'espèce Alsidium $s p$ est la plus répandue des Rhodophycées au niveau de la lagune, où elle occupe environ 55\% de la superficie de la lagune et une fréquence de présence au niveau des échantillons de $42,8 \%$. Sa répartition suit celle des Rhodophycées, avec une concentration plus marquée dans les zones I et II (figure 9). Il semble que Alsidium sp. occupe tous les faciès sédimentaires avec un degré moindre pour les sables vaseux et les sables (tableau 2). Elle est pratiquement présente dans toutes les profondeurs de la lagune à un degré faible dans les fonds dépassant les $6 \mathrm{~m}$.

L'espèce Gracilaria sp occupe 19\% de la superficie de la lagune, avec une fréquence de présence au niveau des échantillons de 19\%. Les Gracilaria sont cantonnés pratiquement dans la moitié nord-ouest de la lagune, avec une répartition spatiale quasi limitée aux deux rives (figure 9). Ces algues ont une tendance à coloniser les vases sableuses et les substrats sablo-vaseux à des profondeurs qui ne dépassent pas $5 \mathrm{~m}$ (tableau 2). Le centre lagunaire n'est pas du tout couvert par les Gracillaires à l'exception d'une aire en face du grand chenal. 


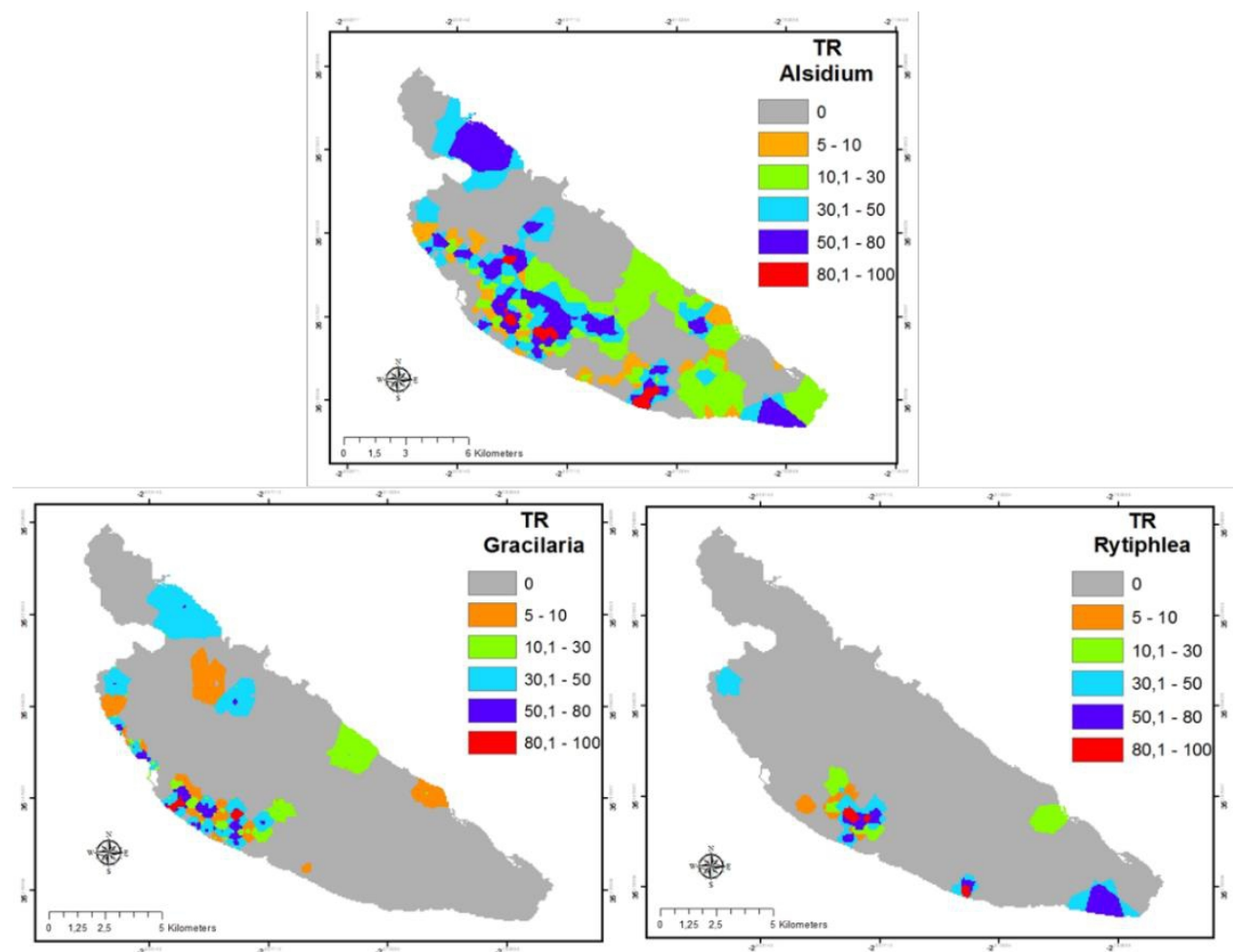

Figure 9 : Répartition spatiale de Alsidium (a), Gracilaria (b) et Rytiphlea (c)

Seule $9 \%$ de la superficie de la lagune est occupée par l'espèce Rytiphlea tinctoria. Elle est en général quasiment présente dans certains endroits localisés de la rive continentale sur des fonds vaseux et sablo-vaseux (figure 9).

\section{Répartition des espèces de Chlorophycées}

19\% des stations échantillonnées renferment Caulerpa prolifera. C'est l'espèce la plus dominante des Chlorophycées, elle occupe une superficie de $25,6 \%$. Ainsi, la tendance de la répartition des chlorophycées suit celle de $C$. prolifera (figure 10). Les zones I et III sont les plus colonisées par cette espèce. Elle se développe sur les différents faciès, à l'exception des sables moyens vaseux et des sables très fins vaseux (tableau 2). Elle est rarement rencontrée au-delà des 5 mètres de profondeur.

L'espèce Chaetomorpha sp. s'étend sur une superficie de $14,8 \%$, avec une fréquence de présence de $6 \%$. Sa répartition est très limitée dans l'espace (figure 10). On la trouve essentiellement au nord-ouest et à certains endroits étroits éparpillés dans la partie nord de la lagune sur des fonds vaseux et sablovaseux. Dans la zone I, la répartition est très étendue sur toute la surface sauf à son extrémité. Les ulves occupent environ une superficie de 5,5\%, avec une 
fréquence de présence de $4 \%$. Ces espèces sont présentes exclusivement sur la rive continentale (zone III) et une partie de la zone I (figure 10). Elles sont rencontrées à des profondeurs faibles ne dépassant pas trois mètres sur des faciès sableux (sables fins, sables moyens vaseux, sables très fins vaseux) et faiblement sur des vases sableuses (tableau 2). L'algue Enteromorpha sp. a été rencontrée au niveau d'une seule station au voisinage des anciens bassins de lagunage, où elle occupe une superficie de $0,1 \%$.

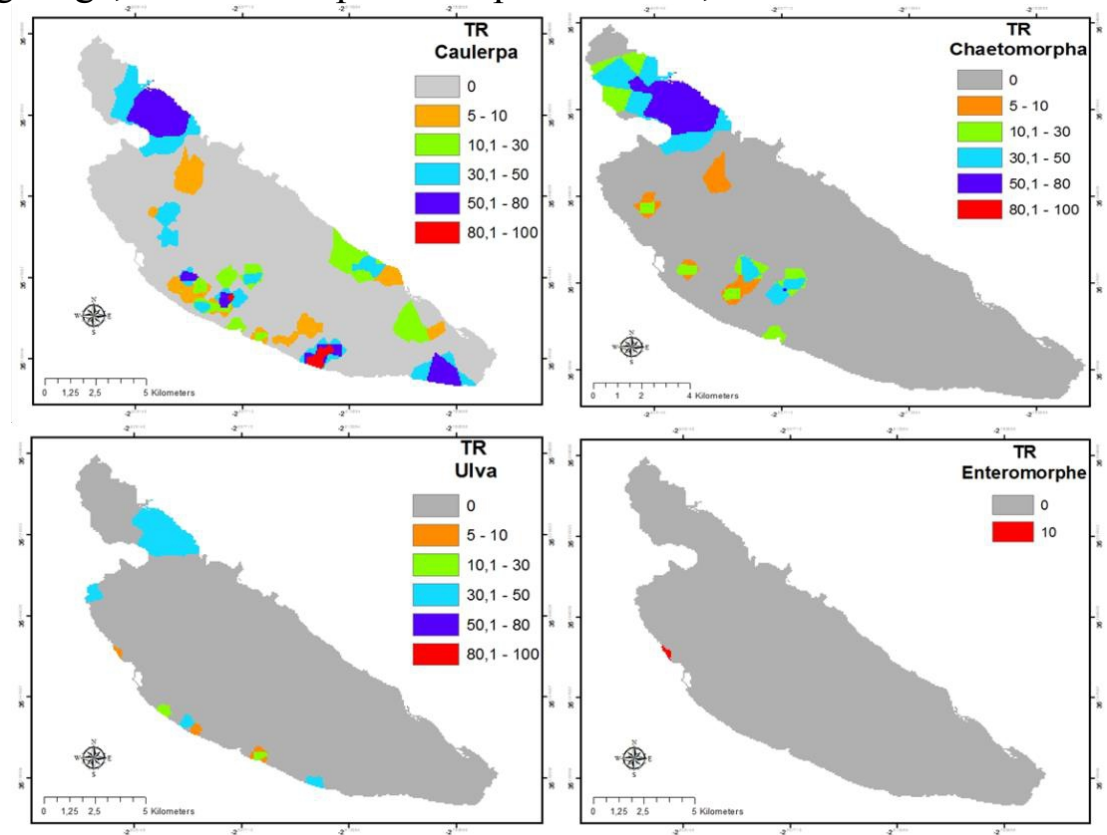

Figure 10 : Répartition Caulerpa (a), Chaetomorpha (b) Ulva (c) et Enteromorphe (d)

\section{Répartition des espèces de Phéophycées}

La présence de Cystoseria sp est très limitée à $0,5 \%$ de la superficie globale, située sur des fonds sableux et vaso_sableux à la rive continentale, au niveau d'un seul point de prélèvement de la zone III (S12) (figure 11).

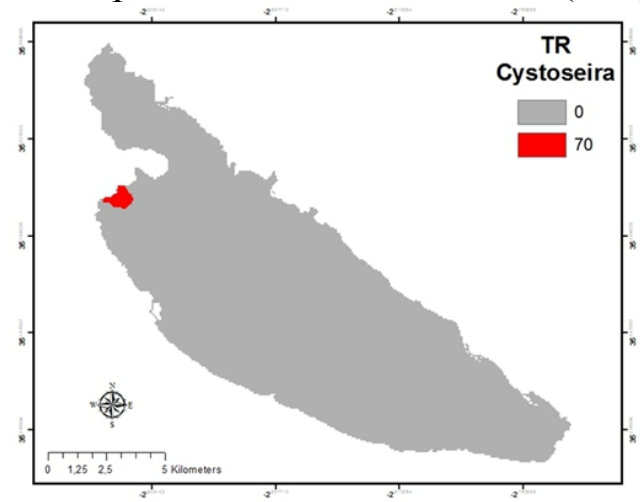

Figure 11 : Répartition de Cystoseia sp.dans la lagune de Nador 


\section{Répartition des espèces de Phanérogames marines}

Les phanérogames marines de la lagune de Nador sont composées de deux genres: Cymodocea sp. et Zostera sp. L'espèce Cymodocea sp. prédomine ces phanérogames et s'étend sur $17,7 \%$ de la superficie totale, contrairement à l'espèces Zostera sp qui occupe seulement 3,7\% de la surface de la lagune et qui reste limitée dans la zone I. Cymodocea sp. est faiblement représentée dans la lagune (figure 12). A l'exception de la zone I, l'espèce est répartie à différents TR exclusivement et partiellement le long des rives.
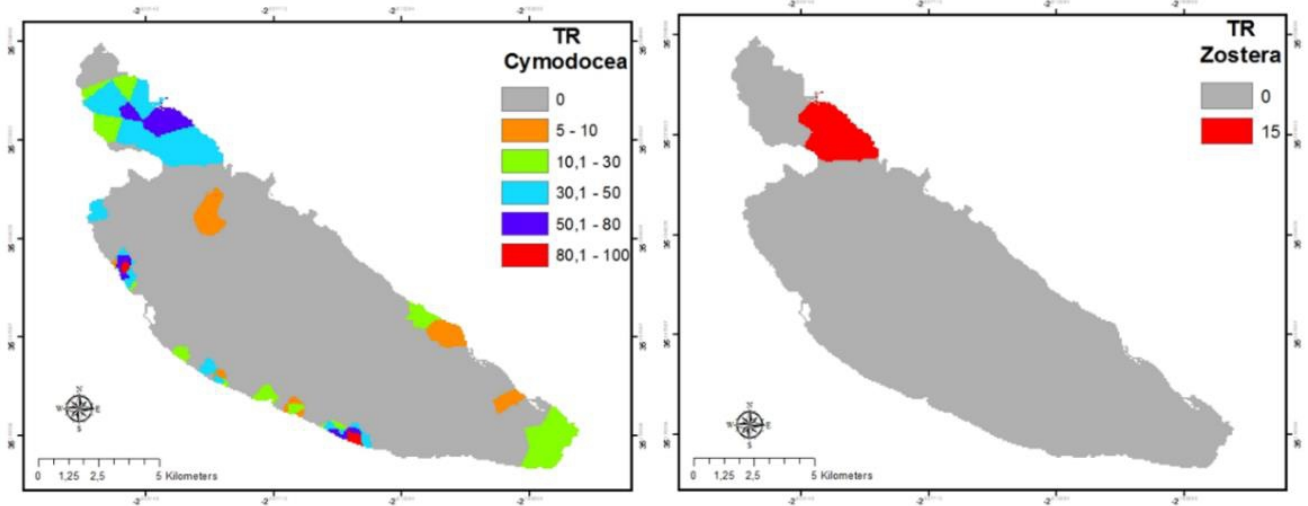

Figure 12 : Répartition de Phanérogames marines au sein de la lagune de Nador

(Cymodocea et Zostera)

La rive du cordon dunaire connait moins de répartition. Elle est rencontrée sur les quatre faciès sédimentaires (tableau 2). Dans la zone I, l'espèce est répartie de la rive jusqu'à $3 \mathrm{~m}$ de profondeur. Sur les deux rives, elle colonise les fonds de faible profondeur, 2 à $3 \mathrm{~m}$ au maximum. Cymodocea sp. est aussi présente au milieu dans la zone IV en face du chenal à des profondeurs allant de 3 à $6 \mathrm{~m}$.

\section{Répartition des taxons selon les caractéristiques physico-chimiques de l'eau du fond}

La macroflore benthique colonise un habitat caractérisé par des paramètres physico-chimiques de l'eau du fond très variables (tableau 3 ). Ainsi, l'habitat est caractérisé par des conditions de température de l'eau de l'ordre de 25 à $29^{\circ} \mathrm{C}$, des salinités de l'ordre de 36 à 38,6g/1 et des teneurs en oxygène dissous de l'ordre de 5 à $9 \mathrm{mg} / \mathrm{l}$. Les teneurs en azote minéral et en orthophosphates sont très variables allant de 5 à $800 \mu \mathrm{g} / 1$ et de 10 à $90 \mu \mathrm{g} / 1$ respectivement.

D'une manière générale, la plupart des espèces de macroalgues et le genre Cymodocea colonisent les zones I et III, caractérisées par des eaux plus riches en nutriments $(\mathrm{N}$ et $\mathrm{P})$. Le genre Alsidium prospère à différentes profondeurs, par contre le genre Gracilaria, les Chlorophycées et le genre Cymodocea se développent dans les endroits moins profonds et ont tendance 
à se concentrer près des rives, probablement pour des raisons photosynthétiques.

Tableau 3: Caractéristiques physico-chimiques de l'habitat des espèces de la macroflore benthique de la lagune de Nador (2011) (Najih et al., Non publié)

Espèce/Genr
Classe $\quad$ Caractéristiques physico-chimiques de l'eau du fond

\begin{tabular}{|c|c|c|c|c|c|c|}
\hline & & $\begin{array}{c}\text { Températu } \\
\text { re } \\
\left({ }^{\circ} \mathrm{C}\right) \\
\end{array}$ & $\begin{array}{l}\text { Salinité } \\
\text { g/l }\end{array}$ & $\begin{array}{c}\text { Azote } \\
\text { minéra } \\
1 \\
\mu \mathrm{g} / 1\end{array}$ & $\begin{array}{c}\text { Orthophospha } \\
\text { tes } \\
\mu \mathrm{g} / 1\end{array}$ & $\begin{array}{l}\text { Oxygè } \\
\text { ne } \\
\text { dissous } \\
\mathrm{mg} / \mathrm{l}\end{array}$ \\
\hline \multirow{3}{*}{ Rhodophycées } & $\begin{array}{l}\text { Rytiphlea } \\
\text { tinctoria }\end{array}$ & $26-28$ & $36-38$ & $\begin{array}{l}5- \\
300\end{array}$ & $10-60$ & $5-9$ \\
\hline & Alsidium $s p$ & $24-29$ & $36-38$ & $\begin{array}{l}5- \\
700 \\
\end{array}$ & $10-90$ & $>5$ \\
\hline & $\begin{array}{l}\text { Gracilaria } \\
s p\end{array}$ & $26-27$ & $37-38$ & $\begin{array}{l}5- \\
300\end{array}$ & $40-90$ & $7-9$ \\
\hline \multirow{4}{*}{ Chlorophycées } & $\begin{array}{l}\text { Caulerpa } \\
\text { prolifera }\end{array}$ & $25-29$ & $36-38$ & $\begin{array}{l}5- \\
800\end{array}$ & $10-90$ & $>5$ \\
\hline & $\begin{array}{c}\text { Chaetomorp } \\
\text { ha sp }\end{array}$ & $26-27$ & $36-38$ & $\begin{array}{l}5- \\
800\end{array}$ & $50-90$ & $>5$ \\
\hline & $\begin{array}{c}\text { Enteromorp } \\
\text { ha sp }\end{array}$ & 25 & 37 & $\begin{array}{l}5- \\
100\end{array}$ & $60-70$ & $7-9$ \\
\hline & Ulva sp & $25-29$ & $>37$ & $\begin{array}{l}5- \\
500\end{array}$ & $30-90$ & $>7$ \\
\hline Phéophycées & $\begin{array}{c}\text { Cystoseira } \\
s p\end{array}$ & 26 & 37,7 & $\begin{array}{l}5- \\
200\end{array}$ & $70-90$ & $>7$ \\
\hline \multirow{2}{*}{$\begin{array}{l}\text { Phanérogames } \\
\text { marine }\end{array}$} & Cymodocea & $26^{\circ}-29$ & $\begin{array}{l}37- \\
38,5\end{array}$ & $\begin{array}{l}5- \\
700\end{array}$ & $10-90$ & 59 \\
\hline & Zoostera & 26 & $37-38$ & $\begin{array}{l}7- \\
500\end{array}$ & $70-80$ & $>7$ \\
\hline
\end{tabular}

\section{Discussion}

Par référence aux travaux de Guelorget et al.(1987) qui ont rapporté que la macroflore benthique tapissait la majeure partie des fonds de la lagune, avec une densité importante durant la période allant de 1980 à 1987 (Figure 13) et aux travaux de Berraho et al.(1995) qui ont permis de montrer que la végétation marine avait disparu dans les localités situées le long de la bordure continentale et à proximité de l'ancienne communication, la présente étude a mis en évidence une régression très poussée du couvert végétal dont la présence reste limitée à environ $64 \%$ de la superficie de la lagune à différentes densités alors que $36 \%$ de son étendue reste dénudée. 


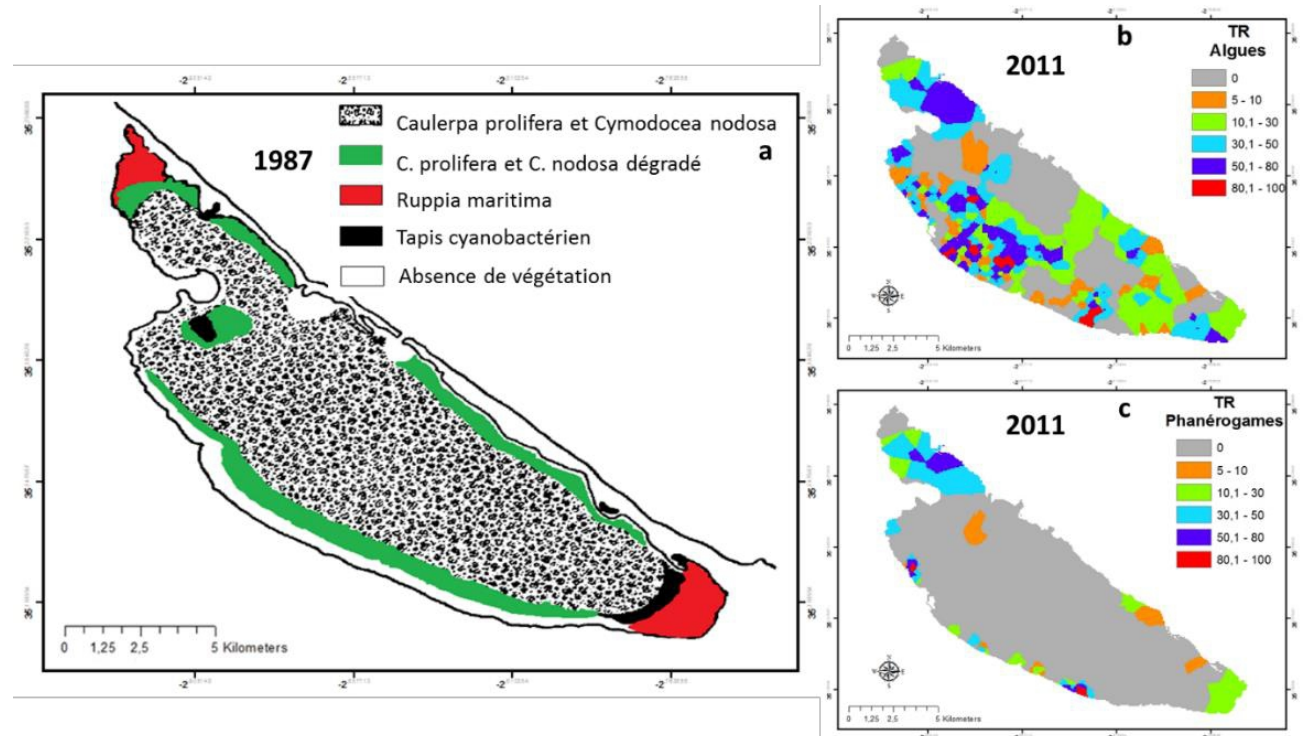

Figure 13 : Répartition spatiale de la macroflore benthique en 1987 (Guelorget, 1987) (a) des macroalgues (b) et phanérogames marines (c) de 2011

L'absence de la macroflorea été observée dans plusieurs localités situées dans les quatre zones de la lagune, avec une absence plus prononcée dans la zone IV et moins prononcée dans la zone III

Il est évident que la nature du sédiment constitue le facteur majeur qui influence d'une manière directe la répartition de la macroflore (Feldman, 1937). Ainsi le sédiment vaso-sableux, qui constituait le faciès majeur des fonds de lagune (Figure2), durant la période de 1987 (Figure 2a), a été probablement à l'origine de la large répartition du couvert végétal en cette période, marquée par une grande dominance des Phanérogames marines et des Caulerpes. Les travaux d'aménagement en 2009 ont conduit à un grand rétrécissement des aires à faciès vaso-sableux et à un envahissement d'une grande partie de la lagune par le faciès vaseux (figure $2 b$ ). Cette modification avait contribué à une nouvelle composition et répartition de la macroflore devenue hautement dominée par les macroalgues, particulièrement les Rhodophycées, ceci au dépend du couvert des Phanérogames marines et des Caulerpales dont l'aire de répartition s'est hautement rétrécie. On note également la disparition de l'espèce Ruppia maritima du fond de la lagune.

Hormis l'effet du faciès sur la répartition de la macroflore benthique, le changement des caractéristiques physicochimiques et hydrodynamiques de l'eau a certainement beaucoup contribué à la modification de cette répartition. En effet, la teneur moyenne en oxygène dissous est passée de $5 \mathrm{mg} / \mathrm{l}$ (Guelorget, 1987) à 9,3mg/ en 2011. La Matière Organique qui dépassait les $20 \%$ en 1987 (Inani, 1997), s'est réduite à moitié $(<10.4 \%)$ en 2011, les teneurs en Azote minéral qui variait de 95 à $1440 \mu \mathrm{g} / \mathrm{l}$ (Berraho et al., 1995) a 
diminué à 5 à $800 \mu \mathrm{g} / \mathrm{l}$ en 2011. Les teneurs en orthophosphates sont passées de 230 à $520 \mu \mathrm{g} / 1$ en 1987 (Berraho et al., 1995) à des valeurs de 10 à $90 \mu \mathrm{g} / 1$ en 2011.Les nouvelles caractéristiques physicochimiques de la lagune sont devenues plus proches de celles de la mer, particulièrement en matière de salinité qui a diminué à des valeurs 37,9 - 38,6g/1 et de $\mathrm{pH}$ qui a augmenté de 7,4 (Inani 1997) à 8,41. Toutefois, une répartition inégale de la macroflore entre la zone IV, moins riche, et la zone III, plus riche, peut être due au degré d'eutrophisation plus élevé au niveau de la zone III sujette à une plus grande influence continentale (tableau 1), favorisant ainsi un enrichissement plus important en nutriments.

L'accentuation de l'hydrodynamisme à travers l'augmentation de la force des courants, a pu, également, contribuer à une diminution du développement de la macroflore par l'arrachement et/ou l'empêchement de la fixation des spores et des jeunes pousses sous la force des courants. Cette hypothèse peut être justifiée par le fait que la zone IV, partie de la lagune la plus exposée aux courants forts engendrés par l'engouffrement des masses d'eau marines, est moins peuplée en macroflore benthique.

Les Rhodophycées restent hautement prédominantes dans le couvert végétal, représentant plus de $58 \%$ de la superficie de la lagune, avec les plus importantes densités enregistrées au niveau de la zone III, ce qui pourrait être expliqué par la richesse de cette zone en nutriments, particulièrement en azote minéral (tableau 1). Les Rhodophycées sont essentiellement représentées par l'espèce Alsidium sp. rencontrée d'une manière régulière dans les quatre parties de la lagune à différentes profondeurs sur des faciès vaseux et vasosableux (tableau2). Tandis que 1'espèce Gracilaria sp. reste cantonnée à de faibles profondeurs dans la partie NO de la rive continentale tout en montrant une répartition régulière. Par contre, elle se répartit d'une manière dispersée dans des localités restreintes de la rive marine sur des faciès vaso-sableux et sablo-vaseux. La comparaison entre les résultats de la présente étude avec ceux obtenus par Id Halla (2004 et 2005) a montré que la surface occupée par Gracilaria a régressé de $54 \%$ à $19 \%$ entre 2005 et 2011 . L'espèce Rytiphlea reste très peu représentée et de faible densité, limitée en majorité à de petites localités de la rive continentale de profondeur $<5 \mathrm{~m}$ sur un faciès de type vaseux et vaso-sableux.

La distribution spatiale des Chlorophycées est irrégulière, se limitant à $32 \%$ de la superficie de la lagune alors que d'anciens travaux ont montré qu'elles se répartissaient le long des rives de la lagune, avec des densités très importantes (Guelorget, 1984, 1986, 1987). En effet, cette répartition s'est rétrécie, suite à l'amélioration de la qualité physico-chimique de l'eau par rapport à la période d'avant l'ouverture du large chenal. D'après Guelorget (1987), les espèces Ulva lactuca et Enteromorpha sp. constituaient un tapis émergeant à la surface de l'eau à la fin du printemps et au début de l'été, 
témoignant d'un certain degré de pollution de l'eau dans ces zones qui a permis leur enrichissement en nutriments ( $\mathrm{N}$ et $\mathrm{P}$ ).

L'espèce Caulerpa proliferaest la plus dominante parmi les Chlorophycées avec un taux d'occupation de 25,7\% de la surface lagunaire. Elle a été rencontrée en association avec Zostera sp. et Cymodocea sp. à des densités variables, mais c'est essentiellement sur des substrat vaseux et sablovaseux que les densités les plus importantes ont été observées avec des TR de l'ordre de 50 à 100\% (tableau2). Toutefois, sa surface d'occupation a connu une grande régression par rapport à la situation décrite par Guelorget (1987) (Figure 13). Des travaux plus récents (Id Halla et al., 2004) ont montré que le processus de régression de l'aire de répartition de Caulerpa n'était pas lié directement aux travaux d'aménagement en 2009, vu qu'il était déjà entamé en 2004,entrainant une réduction de sa surface d'occupation à 47,5\%.

Les Phéophycées restent faiblement représentées au niveau de la lagune de Nador ne couvrant que $11,7 \%$ de la superficie totale. Elles colonisent les quatre types de faciès de la lagune à des profondeurs inférieures à $5 \mathrm{~m}$. L'espèce Cystoseira $s p$. a été rencontrée dans une seule station de prélèvement. Ce taxon est très vulnérable à la pollution et à la turbidité élevée des eaux (c'est un bon indicateur biologique de la qualité de l'eau) dont la présence peut témoigner d'une bonne qualité de l'eau (Belmokhtar, 2012). L'absence de bibliographie sur la répartition de ce taxon, relative à la période précédant 1'aménagement, rend difficile l'affirmation si la biomasse présente est le résultat d'un début de régénération ou d'un processus de disparition suite à son exposition à la pollution le long de la période précédant l'aménagement.

La présente étude a révélé la présence de deux genres de Phanérogames Cymodocea et Zostera d'une répartition très limitée $(18 \%)$. Toutefois l'historique des travaux antérieurs a montré que les fonds de la lagune ont été d'abord couverts par une prairie rase de posidonie à partir de 3 $\mathrm{m}$ de profondeur (Aloncle, 1961). Ces fonds ont été, ensuite, entièrement couvert par des prairies de zostères et de posidonie (Saubade, 1978) et par la suite, les travaux de Guelorget et al., (1983) ont révélé la disparition de la posidonie de l'herbier du fond qui est devenu plutôt représenté par les trois espèces suivantes : Cymodocea nodosa, Zostera marina et Ruppia maritima. Les phanérogames marines semblent avoir plus d'affinité au substrat vasosableux, devenu beaucoup plus réduit après le changement hydrodynamique à partir de 2009. L'espèce Ruppia maritima a également disparu de la lagune en 2011. En effet, cette algue connue comme tolérante au milieu saumâtre a certainement disparu après l'augmentation des masses d'eau marines entrant dans la lagune. 


\section{Conclusion}

$\mathrm{Vu}$ l'absence d'une situation de référence juste avant la mise en œuvre du plan d'aménagement, il est difficile de conclure si les changements qui ont affecté la distribution de la macroflore benthique sont liés aux modifications des facteurs environnementaux de la lagune suite aux travaux d'aménagement. Toutefois, il est fort probable que ces modifications ne peuvent pas rester sans impact sur la macroflore, particulièrement les changements au niveau de la répartition spatiale des nutriments et du faciès, caractérisés par une régression du substrat vaso-sableux, qui semble être bien apprécié par les Phanérogames marines et par une extension du substrat vaseux, qui semble être plutôt bien apprécié par le genre Alsidium qui est devenu le taxon le plus répandu parmi la macroflore benthique de la lagune, atteignant ainsi une surface d'occupation de $55 \%$.

\section{Remerciements}

Les auteurs adressent leurs sincères remerciements à l'équipe du centre régional de l'INRH-Nador pour leur participation aux prélèvements des échantillons, etaux pêcheurs artisans de la lagune de Nador pour leur précieuse collaboration.

\section{References:}

1. Aloncle H. 1961. La pêche dans la Marchica de Mellila. Bull. Inst. Pêches Maroc, $7:$ 13-22.

2. Belmokhtar, 2012) Cystoseira amentacea : indicateur de la qualité des eaux côtières de l'ouest algérien. Thèse Magister Université d'Oran Es-Senia 65p.

3. Berraho A., A. Orbi et J.E. Dafir, (1995) : La lagune de Nador : organisation, fonctionnement et évolution. Travaux et Documents $\mathrm{N}^{\circ}$ 85, Institut Scientifique des Pêches maritimes 66p.

4. Canfield, 1942 Canfield, R. H. 1942 : Sampling ranges by the line intercept method : plant cover, composition, density, and degree of forage use. Res. Rep. 4. Tucson, AZ: U.S. Department of Agriculture, Forest Service, Southwestern Forest and Range Experiment Station. 21 $\mathrm{p}$

5. (Dakki, 2003) Diagnostic pour l'aménagement des zones humides du Nord-Est du Maroc - Sebkha bou Areg (Lagune de Nador). MedWetCoast-Maroc. Ministère de l'Aménagement du Territoire, de l'Eau et de l'Environnement-Secrétariat d'Etat à l'Environnement. Département des Eaux et forêts et de la Lutte contre la Désertification.

6. Feldman (1937). Recherches sur la végétation marine de la Méditerranée. La côte des Albères. Revue Algologique 10, 73-254 
7. Fischer, W., M.-L. Bauchot and M. Schneider (eds.), 1987. Fiches FAO d'identification des espèces pour les besoins de la pêche. (Révision 1). Méditerranée et mer Noire. Zone de Pêche 37. FAO, Rome. 1529 p.

8. Guelorget O. et Perthuisot J. P., 1983. Le domaine paralique : Expressions géologiques, biologiques et économiques du confinement. Presse de l'Ecole Normale Supérieure, Série 156, 16, 128p.

9. Guelorget O., Perthuisot J.P., Frisoni G.F. \& et Monti D.1987. Le rôle du confinement dans l'organisation biogéographique de la lagune de Nador (Maroc). Oceanologica Acta, 10 (4) : 435-444.

10. Guelorget O., Monti D., Frisoni G.F. et Perthuisot J.P. 1984. Diagnose écologique de la lagune de Nador. Rapp. FAO, mai 1984, Ronéo, 52 p.

11. Himi K., Makaoui A., Idrissi M., Abdellaoui B., El Ouehabi Z. (2015). Circulation marine de la lagune de Nador (Maroc) par modélisation hydrodynamique. European Scientific Journal November 2015-edition vol.11, No.32 ISSN: 1857 - 7881 (Print) e - ISSN 1857- 7431].

12. Hilmi k. (2005) Modélisation numérique de la circulation de deux milieux paraliques du littoral marocain: la lagune de oulidia (atlantique) et la lagune de Nador (méditerranée) thèse pour l'obtention du diplôme de doctorat faculté des sciences ben m'sick.

13. Id Halla et B. Abdellaoui (2005 ) Gracilaria et Gélidiu dans la lagune. Rapport interne 30p.

14. Id Halla et B. Abdellaoui '2004) PROSPECTION ET EVALUATION DE LA BIOMASSE DES AGAROPHYTES (Gracilaria sp. et Gélidium sp.) DANS LA LAGUNE DE NADOR. Rapport interne $28 \mathrm{p}$.

15. Inani I. (1995). Dynamique sédimentaire et état de pollution dans la lagune de Nador. Thèse de 3ème cycle. Université Med V Agdal Rabat, 186p et 37 figures.

16. Lefebvre A., Guelorget O., Perthuisot J.P. et Dafir J. (1997): Évolution biogéologique de la lagune de Nador (Maroc) au cours de la période 1982-1993. Oceanologica Acta, 20, 2, pp. 371-385.

17. Najih M, Nachite D., Berday N., Pastres R., Lamrini A. Rezzoum N. (2016). Charactérization of the new status of Nador lagoon (Morocco) after the implementation of the management plan. Journal of Marine Science and Engineering. Article sous press.

18. Re V. (2010) Groundwater in urban coastal areas: Hydrogeochemical based approach for managing the transition areas. The example of the lagoon of Nador (Morocco). PhD Programme in Analysis and Governance of Sustainable Development Universita Ca'Foscar Venezia 153p. 
19. Saubade A. M. 1979. la malacofaune actuelle (bivalves et gastéropodes de la lagune de Nador, côte méditerranéenne du Maroc. Bull. Inst. Géolo. Du bassin d'Aquitaine, Bordeaux 26. 1-89.

20. Zine N., (1989) : Etude de la malacofaune de la lagune de Nador et dynamique de population de Venerupis decussata (Linné 1767). Thèse doct. 3ème cycle, Fac. Sci. Rabat, 97 pp. 